Limnetica, 26 (2): 359-372 (2007). DOI: 10.23818/limn.26.31

(c) Asociación Ibérica de Limnología, Madrid. Spain. ISSN: 0213-8409

\title{
Protocolo para la evaluación del estado ecológico de la red fluvial de Aragón (NE de España) según sus comunidades de macroinvertebrados bentónicos
}

\author{
Antonio Torralba-Burrial * y Francisco J. Ocharan \\ Departamento de Biología de Organismos y Sistemas. Universidad de Oviedo. E-33071 Oviedo, España \\ * Corresponding author: antoniotb@hotmail.com
}

\begin{abstract}
Protocol to evaluate the ecological status of the fluvial network of Aragón (NE of Spain) according to the benthic macroinvertebrate communities

Rivers play a fundamental role in the biosphere, and to attain a sustainable development it is necessary to evaluate their ecological status, to know how and which human activities affect them, the Water Framework Directive (2000/60/CE) establishes the basic principles for a sustainable water use policy in the EU. With the aim of evaluate the ecological status of the fluvial network of Aragón for the Aragon Government, a protocol for sample taking and analysis was designed to cover two objectives. It had to produce enough precise results and also, it had to allow for space-time comparisons, in accordance with the Directive and international standardization rules. It is based on sampling with Surber net in laminar and turbulent flow, IBMWP index applications, evaluation of the differences in abundance of macroinvertebrates, evaluation of the presence of alien species, assignation of ecological state categories, and cartographic representation. Through this protocol, the ecological status of 140 reaches of the Aragon fluvial network was determined. Abundance corrections were not necessary and only one correction was due to the presence of alien species.
\end{abstract}

Key words: Ecological status. Rivers. Aragón. Iberian Peninsula. Benthic macroinvertebrates. Protocols. Water Framework Directive.

\section{RESUMEN}

Protocolo para la evaluación del estado ecológico de la red fluvial de Aragón (NE de España) según sus comunidades de macroinvertebrados bentónicos

Los ríos desempeñan un papel fundamental en la biosfera y para conseguir un desarrollo sostenible es necesario valorar su estado ecológico, comprobando qué actuaciones humanas les afectan y cómo; la Directiva Marco de Aguas (2000/60/CE) establece los principios básicos para una política hídrica sostenible en la UE. Con el objeto de valorar el estado ecológico de la red fluvial aragonesa para el Gobierno de Aragón, se diseñó un protocolo de toma y análisis de muestras que cubriera dos objetivos. Debía producir unos resultados suficientemente precisos y, además, debía permitir las comparaciones espaciotemporales, siguiendo tanto la Directiva como las normas internacionales de estandarización. Está basado en el muestreo con red Surber tanto en flujo laminar como turbulento, aplicación del índice IBMWP, evaluación de las diferencias en la abundancia de macroinvertebrados, valoración de la presencia de especies alóctonas, asignación de categorías de estado ecológico y representación cartográfica. Mediante este protocolo se determinó el estado ecológico de 140 tramos de la red fluvial aragonesa, no siendo necesarias correcciones por abundancia y sólo una corrección por la presencia de especies alóctonas.

Palabras clave: Estado ecológico. Ríos. Aragón. Península Ibérica. Macroinvertebrados bentónicos. Protocolos. Directiva Marco de Aguas. 


\section{INTRODUCCIÓN}

Los ríos han sufrido contaminaciones y otras agresiones antrópicas desde tiempos históricos, a pesar de que desempeñan un papel fundamental en el ciclo hidrológico global, proporcionando importantes servicios a los humanos y contribuyen de forma importante a mantener la biodiversidad. La Directiva Marco del Agua (Directiva 2000/60/CE; DMA), establece los principios básicos para una política hídrica sostenible en la Unión Europea. Esta Directiva tiene como objeto fijar un marco para la protección de las aguas que prevenga todo deterioro adicional y proteja y mejore el estado de los ecosistemas acuáticos, refiriendo principalmente este objetivo a la calidad de las aguas afectadas.

El estado ecológico de las aguas superficiales debe entenderse como una expresión de la calidad de la estructura y el funcionamiento de los ecosistemas acuáticos asociados a las mismas. Este estado ecológico se expresará en función de cinco categorías: muy bueno, bueno, aceptable, deficiente y malo, definidas en el Anexo V de dicha Directiva.

La Directiva establece los indicadores de calidad para la clasificación del estado ecológico de las masas de agua (ríos, lagos, aguas de transición...). El estado ecológico se determina a partir de indicadores biológicos, indicadores hidromorfológicos que afectan a los indicadores biológicos e indicadores químicos y fisicoquímicos que afectan a los indicadores biológicos, lo que nos da una idea de la importancia que tienen los indicadores biológicos en la Directiva. Dentro de los indicadores biológicos para ríos recoge: 1/composición y abundancia de la flora acuática, 2/composición y abundancia de la fauna bentónica de invertebrados, y 3/composición, abundancia y estructura de edades de la fauna ictiológica.

Teniendo presente esa necesidad de valorar el estado ecológico de los ríos aragoneses, la Dirección General del Medio Natural del Gobierno de Aragón puso en marcha, en el año 2001, la elaboración de un protocolo que permitiría estandarizar el procedimiento para valorar el estado ecológico de los ríos según las comunidades de invertebrados bentónicos.

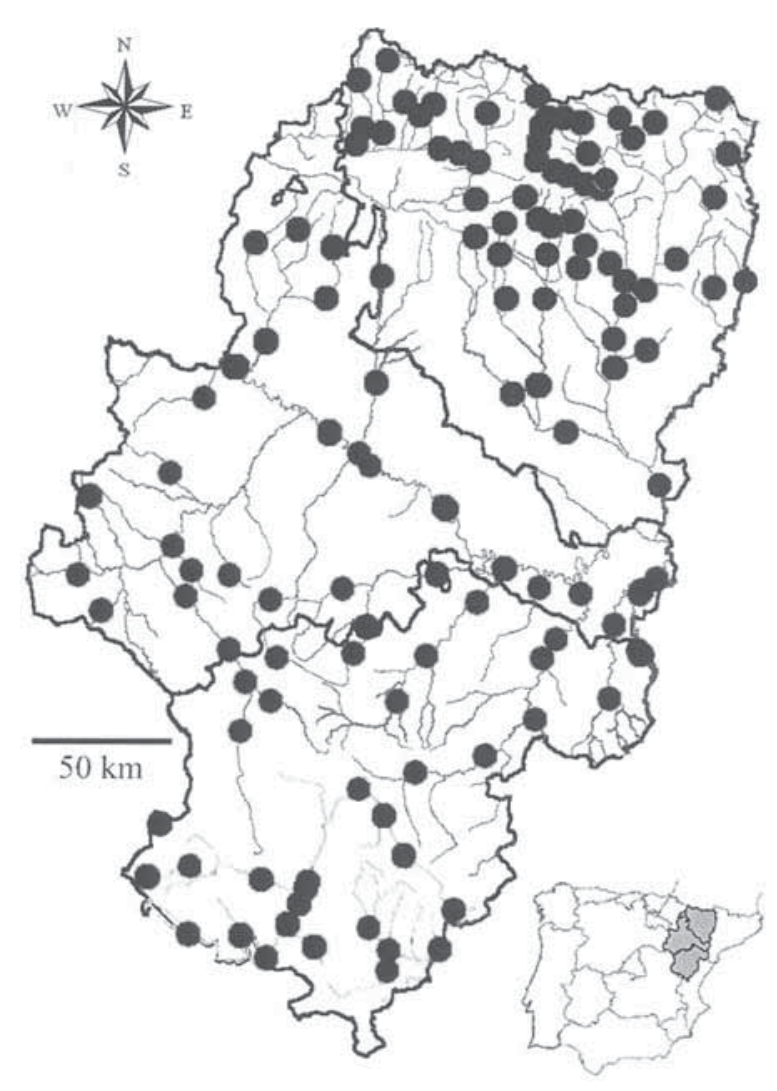

Figura 1. Situación de los puntos muestreados en la red fluvial aragonesa. Sampling site locations in the fluvial network of Aragon.

Además, dicho protocolo debía evaluarse en los distintos tipos de ríos presentes en Aragón.

En los años anteriores se estaban empleando numerosos sistemas de muestreo e interpretación de datos para valorar la calidad de las aguas mediante el estudio de las comunidades de macroinvertebrados. Actualmente el protocolo GUADALMED (Jáimez-Cuéllar et al., 2002) ha sido aplicado en numerosas cuencas mediterráneas peninsulares. Sin embargo, en el reciente V Congreso Ibérico de Limnología, con varias sesiones temáticas dedicadas a "la Directiva Marco del Agua: protocolos de estado ecológico" se pudo observar que continuaba la falta de homogeneidad metodológica.

Teniendo en cuenta las indicaciones de la DMA, y la diversidad de protocolos existentes en la fecha del estudio, los objetivos de este trabajo son: 1) diseñar un protocolo de toma y análisis de muestras 


\section{Protocolo: campo}

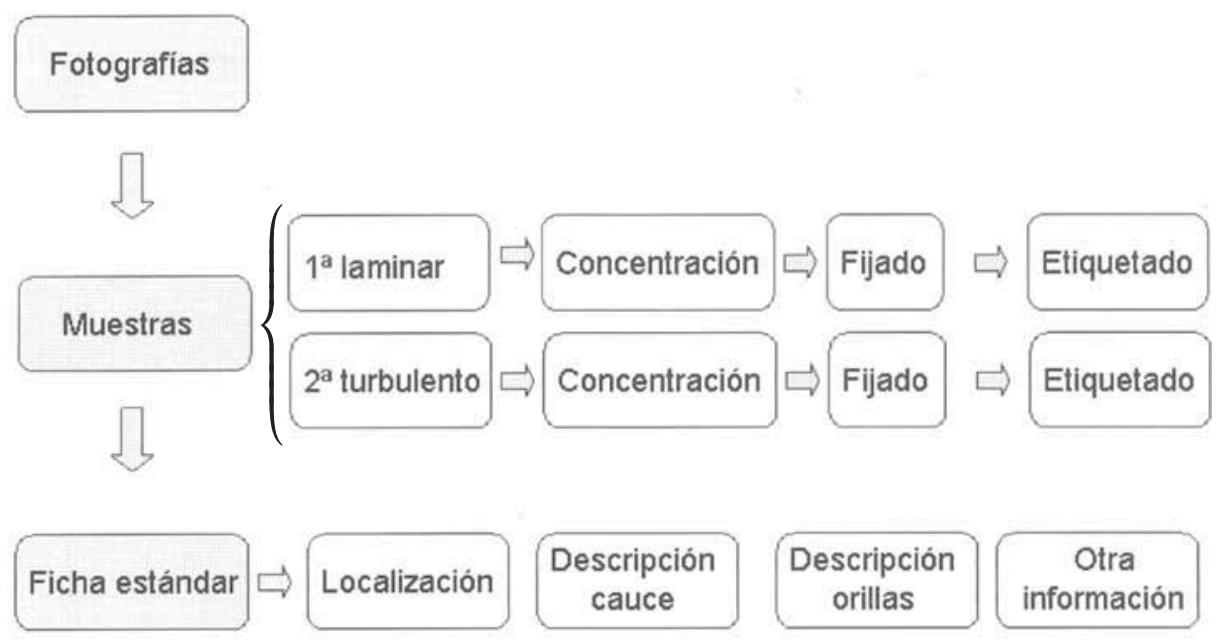

Figura 2. Esquema del protocolo a seguir en los trabajos de campo. Protocol scheme to be used in field work.

compatible con la DMA; y 2) evaluar el estado ecológico de la red fluvial de Aragón (NE de España).

\section{MÉTODOS}

En el Apartado 1.3.6 del Anexo V de la DMA se recogen diversas indicaciones sobre los protocolos a emplear. En dicho apartado se especifica que los métodos utilizados para medir cada parámetro serán conformes a normas (internacionales o nacionales) que garanticen la obtención de una información de calidad y científicamente comparable. Esto es, siguiendo la DMA, un protocolo debería: 1) obtener resultados suficientemente precisos, 2) permitir comparaciones espacio-temporales, y 3) seguir las normas internacionales de estandarización y la propia DMA. En el caso concreto de los macroinvertebrados bentónicos, se especifican las normas internacionales de tipificación en las que deben basarse los sistemas de recogida de dichos organismos. Se han consultado y valorado asimismo otros protocolos empleados en el resto del mundo (Klemm et al., 1990; Lenat \& Barbour, 1994; Plotnikoff, 1994; Resh et al., 1995; Barbour et al., 1996, 1999; Rosenberg et al., 1997), de los cuales se han empleado aquellos aspectos que se han considerado interesantes, en aras de buscar la mayor estandarización posible, siempre y cuando fueran compatibles con los requerimientos establecidos por la Directiva 2000/60/CE.

El protocolo resultante se puso a prueba y se empleó para evaluar el estado ecológico de los ríos aragoneses según sus comunidades de macroinvertebrados bentónicos. En 2001 y 2002 fueron muestreados siguiendo este protocolo 140 tramos repartidos por toda la red fluvial aragonesa (Fig. 1). Estos puntos se eligieron con el fin de que abarcaran la mayoría de las cuencas y tipos de ríos presentes en Aragón para comprobar la aplicabilidad del protocolo en todo el territorio.

\section{RESULTADOS}

\section{Protocolo}

El material necesario para llevar a cabo este protocolo se indica en la Tabla 1.

\section{Trabajo de campo}

Las actuaciones del protocolo en el río se esquematizan en la figura 2 . 
Tabla 1. Equipo y material necesarios para llevar a cabo el protocolo. equipment and material necessary to carry on the protocol.

\begin{tabular}{|c|c|}
\hline Equipo & Especificaciones \\
\hline Muestreador Surber & $\begin{array}{l}\text { Está constituido por dos bastidores cuadrados ( } 30 \mathrm{~cm} \text { lado), articulados uno sobre otro, llevando } \\
\text { uno de ellos la red y definiendo el otro el área de recogida. } 250 \mu \mathrm{m} \text { de luz de malla evitan la } \\
\text { perdida de pequeños taxones (EN 28265:1994) }\end{array}$ \\
\hline Bandeja de muestreo & $\begin{array}{l}\text { De plástico, por su bajo peso y poco riesgo de rotura. Deberá ser lo suficientemente amplia como } \\
\text { para permitir el vaciado de la red en ella, pero por motivos prácticos no deberá ser demasiado } \\
\text { grande. Unas medidas orientativas serían } 30 \times 20 \times 6 \mathrm{~cm} \text {. Colores claros, especialmente el blanco, } \\
\text { facilitan la localización de los invertebrados en la bandeja. }\end{array}$ \\
\hline Botes para muestras & $\begin{array}{l}\text { De plástico, cilíndricos (boca de una tamaño similar al recipiente en sí), con cierre hermético, } \\
\text { de tapa a rosca y obturador, y de } 500 \mathrm{ml} \text { de volumen. Presentan bajo riesgo de rotura, facilidad } \\
\text { de cierre hermético y de reapertura, tamaño y forma adecuada para las muestras que se deben } \\
\text { tomar, baja masa, fácil disponibilidad, bajo coste y facilidad de limpieza y reutilización. }\end{array}$ \\
\hline Filtro & $\begin{array}{l}\text { Facilita la concentración de la muestra y su traspaso al bote, } 200 \mu \mathrm{m} \text { de luz de malla (inferior a } \\
\text { la de la red) para evitar la pérdida de los individuos de menor talla o incompletos. }\end{array}$ \\
\hline Garrafa plástica & Para el etanol. De volumen adecuado al número y tipo de muestras a recoger. \\
\hline Papel etiquetas & $\begin{array}{l}\text { No deben utilizarse papeles de baja densidad ni que tiendan a disgregarse con facilidad. El papel } \\
\text { vegetal o la cartulina dan buenos resultados. }\end{array}$ \\
\hline Rotuladores punta fina & $\begin{array}{l}\text { Para el etiquetado exterior de los botes deben se resistentes al agua y sol (lo que permite su } \\
\text { almacenaje, limpieza y posterior reutilización); para el interior resistentes también al alcohol. } \\
\text { Conveniente llevar más de uno de cada tipo. }\end{array}$ \\
\hline Pinzas finas & Facilitan la recogida de ejemplares adheridos a la red. \\
\hline Microscopio estereoscópico & Para la determinación de los macroinvertebrados en el laboratorio. \\
\hline Tubos o botes & $\begin{array}{l}\text { Para conservación de muestras, cierre hermético para evitar la evaporación del líquido } \\
\text { conservante }\end{array}$ \\
\hline Claves determinación & Hasta el nivel taxonómico requerido (familia principalmente) (p. ej. Tachet et al. 2000) \\
\hline
\end{tabular}

Prevención de riesgos

A la hora de realizar los trabajos de campo, es recomendable tener en cuenta ciertas medidas de seguridad. Así, no es prudente que una persona trabaje individualmente en el agua debido a los riesgos originados por las velocidades elevadas de corrientes, aguas profundas y lechos inestables, a lo que hay que añadir posibles cambios bruscos en el caudal, bien de forma natural o producidos por las sueltas de las centrales hidroeléctricas. Se recomienda por tanto la presencia de dos personas en los muestreos. Se deben usar guantes para proteger las manos de la abrasión que pueden provocar las piedras del lecho del río, además de evitar posibles cortes por cristales u otras basuras. En el caso de que el agua esté muy fría (ríos de montaña...), se recomienda usar guantes que aíslen de esa frialdad (p. ej. de neopreno). Igualmente puede ser necesario el uso de botas altas de goma o de un vadeador por los mismos motivos.

\section{$\underline{\text { Caracterización de la estación }}$}

Una vez en el tramo a muestrear, es recomendable tomar una o varias fotografías, antes de penetrar en el lecho del río, que posteriormente pueden ayudar en la descripción del mismo y de las condiciones de la corriente.

Se deberá rellenar una ficha o formulario de cada lugar muestreado. Esta ficha contendrá los datos recogidos en la etiqueta, siendo complementados por las observaciones que se consideren pertinentes (cobertura y tipo de la vegetación sumergida, contaminación aparente, caudal anormal, presencia de especies alóctonas...). Se deberá rellenar este formulario in situ, inmediatamente después de tomar la muestra. 
Toma de muestras

Tras elegir el primer punto de muestreo, en flujo laminar, se sumerge el muestreador en el agua con los bastidores abiertos en ángulo recto, dirigiendo la extremidad abierta aguas arriba, de forma que la red queda abierta a la corriente. Se coloca firmemente el bastidor inferior sobre el suelo, con el fin de delimitar el área de recogida. Se remueve y lava el contenido del bastidor inferior en la red, hasta una profundidad de entre 30 y 100 mm, según el sustrato. En el caso de que no haya suficiente corriente, es necesario generarla con la mano para que los invertebrados entren en la red. Los técnicos que recogen la muestra deben evitar rigurosamente situarse aguas arriba de la boca de la red. Así se evita que sus pisadas levanten invertebrados que acabarían en la red sin estar dentro del área de muestreo. Del mismo modo, no debería haber otras personas u otros animales que pudieran levantar a los macroinvertebrados aguas arriba de la red.

Posteriormente se vierte lo recogido sobre la bandeja, se lavan en ella las piedras y ramitas y se concentra y pasa la muestra a un bote ayudados por el filtro. Se fija, etiqueta y conserva la muestra según el siguiente apartado.

Posteriormente se toma una segunda unidad muestral en flujo turbulento, repitiendo el proceso, que complementará a la anterior pudiendo recolectar taxones no presentes en el otro tipo de flujo.

Conservación y etiquetado

La muestra debe ser fijada inmediatamente con alcohol etílico a una concentración superior al $70 \%, 96 \%$ en el caso de que contenga una cantidad considerable de algas o sedimentos (si hay muchos puede ser necesario utilizar más de un bote para garantizar su correcta fijación). Es importante que el volumen de líquido fijador sea mayor que el de la muestra a fijar, o de lo contrario puede iniciarse el proceso de descomposición de los invertebrados antes de llegar al laboratorio.

$\mathrm{El}$ bote se debe etiquetar internamente en ese mismo momento, de un modo claro y duradero para permitir su identificación en el laboratorio sin ninguna ambigüedad. Una rotulación externa adicional facilita la localización de cada muestra. Los datos que deben aparecer en la etiqueta son: río, localidad (provincia), fecha, y nombre del técnico que recoge la muestra. Se deberá identificar sin posibilidad de confusión el lugar exacto en el que se ha tomado la muestra, recomendándose el empleo de un GPS.

\section{Trabajo de laboratorio}

Se lleva la muestra al laboratorio, esquematizándose su determinación y análisis en la figura 3.

\section{Control de las muestras}

Se recomienda contrastar el número de muestras que llega al laboratorio con el número de muestras recogidas en el campo, con el fin de confirmar que no se han producido pérdidas en el transporte.

Igualmente es aconsejable contrastarque el etiquetado interior y exterior de cada muestra es el mismo.

Determinación y recuento

Se procederá a la determinación y recuento de los invertebrados, principalmente hasta el nivel taxonómico de familia, que es el recomendado en la normativa internacional (EN ISO 8689-1). Se etiquetarán y conservarán las muestras en alcohol de $70^{\circ}$, en recipientes adecuados que eviten la evaporación del líquido conservante.

Evaluación del estado ecológico

Con los taxones presentes en las dos unidades muestrales de cada tramo se aplicará la valoración del IBMWP. Si nuevos estudios no indican lo contrario, se considerarán las cinco clases originales del IBMWP.

Se procederá a continuación a valorar la abundancia de los macroinvertebrados bentónicos presentes en la comunidad, por si hubiera disminuciones importantes asociada a presiones humanas sobre el cauce. Esta valoración se hará teniendo en cuenta los datos existentes, y se aplicará sólo cuando la disminución en las densidades sea muy elevada y no achacable a condicionantes naturales (p. ej., riadas).

A continuación se valorará la presencia de especies alóctonas en la comunidad (datos ex- 


\section{Protocolo: laboratorio}

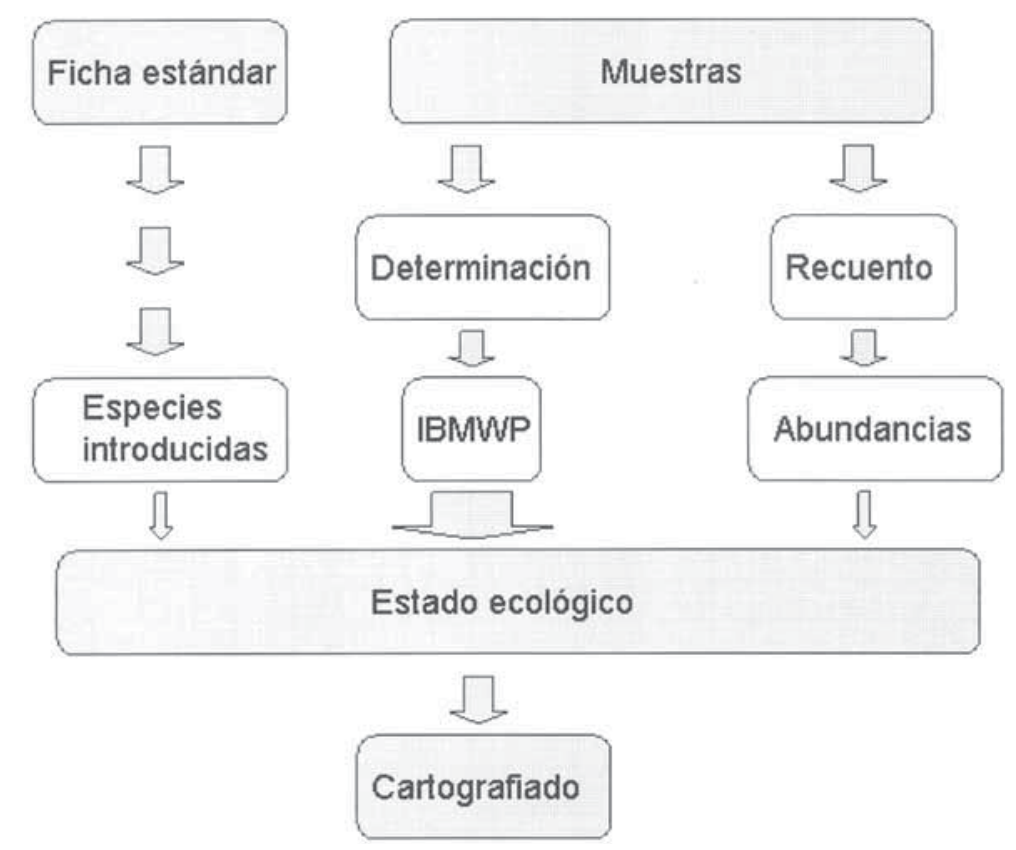

Figura 3. Esquema del protocolo a seguir en los trabajos de laboratorio. Protocol scheme to be used in laboratory work.

traídos de la ficha de campo), ya que representan una alteración de origen humano en la composición del ecosistema, en ocasiones de fatales consecuencias. Sólo consideramos aquí aquellos invertebrados que pueden afectar negativamente a invertebrados autóctonos recogidos en los Catálogos Nacional y Aragonés de Especies Amenazadas y que han sido encontrados en Aragón, por considerar que el efecto global sobre la comunidad nativa ya resulta valorado mediante los puntos anteriores. La penalización aplicada se considera proporcional al impacto que pueden causar sobre esas especies catalogadas (Tabla 2). Esto es, si una estación mantiene una comunidad de macroinvertebrados bentónicos que, aplicando la valoración del IBMWP, le otorgaría una clase I, pero está presente el cangrejo introducido Procambarus clarkii, se penaliza esta alteración antrópica que impide la presencia de especies catalogadas (en este caso Austropotamobius pallipes), disminuyendo la clase de estado ecológico asignada a II. $\underline{\text { Representación de los resultados }}$

El cartografiado de las estaciones seguirá el código de colores indicado en la DMA: azul para el estado ecológico muy bueno, verde para el bueno, amarillo para el moderado, naranja para el deficiente y rojo para el malo.

\section{Aplicación}

Siendo este un trabajo metodológico no nos extenderemos en exponer y discutir aquí el estado ecológico de la red fluvial de Aragón, sino que solo indicaremos cómo fue aplicado el protocolo. Las comunidades de zonas de cabeceras no alteradas, incluso los tramos inferiores menos perturbados, alcanzaron valores en la puntuación del IBMWP superiores a 100, siendo en algunos casos superiores a 250, incluso en ríos mediterráneos.

No fue necesario realizar ningún cambio de categoría debido a la abundancia de los taxones; aquellas estaciones con densidades muy inferiores a las de las estaciones poco alteradas 
Tabla 2. Especies invasivas presentes en Aragón que afectan a especies incluidas en los Catálogos Nacional o de Aragón de Especies Amenazadas, taxones catalogados a los que amenazan y clase máxima de estado ecológico que asignaremos a un tramo afectado por la presencia de estos organismos. Invasive species present in Aragon that affect species included in the National and Aragon Endangered Species Lists, catalogued taxa that are threatened, and maximal class of ecological status assigned to a reach affected by the presence of these organisms.

\begin{tabular}{|ccc|}
\hline Especies invasivas & Especies catalogadas afectadas & Clase máxima estado ecológico \\
\hline $\begin{array}{c}\text { Procambarus clarkii } \\
\text { Pascifastacus leniusculus }\end{array}$ & Austropotamobius pallipes & II \\
\hline $\begin{array}{c}\text { Dreissena polymorpha } \\
\text { Corbicula fluminea }\end{array}$ & Margaritifera auricularia & III \\
\hline
\end{tabular}

presentaron también puntuaciones más bajas según la valoración del IBMWP, con lo que ya habían sido adscritas a una clase inferior.

En el caso de las especies introducidas, sólo hubo una estación que presentara valores del IBMWP propios de una clase I y en la que al mismo tiempo fuera detectada la presencia de una especie invasiva (abundancia elevada de Procambarus clarkii). Se trató del río Cinca en Enate. En el resto de tramos la presencia de especies alóctonas iba asociada a valores inferiores del IBMWP.

Los ríos pirenaicos, los prepirenaicos, la mitad de los tramos de los grandes ríos y el $85 \%$ de los situados en el Sistema Ibérico mostraron un estado ecológico muy bueno o bueno. El estado ecológico fue inferior en el $16 \%$ de los tramos: parte baja de grandes ríos, Turia aguas abajo de Teruel... identificándose importantes perturbaciones. Resultan tristemente llamativos los bajos valores de los índices obtenidos en el río Ebro, especialmente aguas abajo de Zaragoza.

\section{DISCUSIÓN}

\section{Método muestreo}

La selección de un método de muestreo determinado es probablemente una elección difícil para establecer un sistema de evaluación biológica rápido (Lenat \& Barbour, 1994), y entre otras cosas acaba dependiendo del tipo de datos que queremos obtener. En este caso se debía elegir uno de los métodos indicados por la DMA.

Para las comunidades de macroinvertebrados en aguas fluviales someras, la DMA re- mite a las normas de muestreo con red manual (EN 27828:1994) o con muestreador Surber (EN 28265:1994), añadiéndose otros sistemas para aguas profundas (EN ISO 9391:1995). Comparaciones anteriores de ambos métodos, incluyendo la propia norma de estandarización de la red manual, muestran una elevada correlación entre los resultados obtenidos con ambos métodos de muestreo, capturando habitualmente un mayor número o igual de taxones el muestreador Surber frente a la red manual (Storey et al., 1991; Genomi \& Strada, 2000).

Con el fin de confirmar esta correlación en la zona de estudio, realizamos una comparación en 15 estaciones que abarcaban la mayor parte de tipos de ríos y estado ecológico presentes en Aragón, empleando el máximo tiempo recomendando en la norma de estandarización de la red manual (10 minutos) y dos Surber (en distintos flujos) (Torralba Burrial \& Ocharan, 2007). Ningún método de muestreo puede garantizar la captura de todos los taxones presentes en un tramo, pero el comparar los resultados de métodos distintos nos permite evaluar su fiabilidad relativa. Los resultados muestran la gran correlación existente entre ambos métodos en el número de taxones capturados $(a=1.07$; $r=0.90$ ), número de familias EPT (Ephemeroptera, Plecoptera y Trichoptera) $(a=0.98$; $r=0.94)$ y número de taxones que entran a formar parte del índice IBMWP $(a=1.08 ; r=0.93)$. De aplicar la valoración del IBMWP, también existe una alta correlación en su valor $(a=1.10$; $r=0.95)$ y en el del IASPT $(a=0.90 ; r=0.92)$. Pese a estas elevadas correlaciones, el muestreador Surber, combinando los datos de ambos flujos, presenta una mayor eficiencia de cap- 
tura, recolectando un número ligeramente mayor de taxones (Torralba Burrial \& Ocharan, 2007).

Además, el muestreador Surber proporciona datos numéricos de abundancias, expresables como densidades (número de individuos por metro cuadrado). Esto, pese a ser muy conveniente, no lo podemos conseguir con la red manual, por lo que la comparación se decanta a favor del muestreador Surber. Y la posibilidad de obtener estas estimas de la abundancia de los invertebra- dos es una ventaja importante de la utilización de este protocolo frente a otros.

Muestreos con Surber han sido ampliamente empleados para valorar la calidad de las aguas, incluyendo su uso en la Península Ibérica con valoraciones del BMWP original (Younes et al., 2002), RIVPACS basado en él (Armitage et al., 1990) e IBMWP (Tabla 3). Este tipo de muestreo no es aplicable en ríos muy profundos, ya que requiere que el operador se encuentre

Tabla 3. Técnicas de muestreo en las que se ha empleado o propuesto la valoración del IBMWP. Sampling techniques in which IBMWP scores have been used or proposed.

\begin{tabular}{|c|c|}
\hline Referencia & Tipo muestreo \\
\hline Alba-Tercedor \& Jiménez Millán 1985 & 2 ó 3 Surber (zonas de orilla y más centrales) \\
\hline Pujante et al. ,1995 & $\begin{array}{l}\text { transecto de } 25 \mathrm{~m} \text { y hasta el centro de la corriente con Surber de } 2 \mathrm{~mm} \text { de } \\
\text { luz de malla }\end{array}$ \\
\hline $\begin{array}{l}\text { Alba-Tercedor 1996; Alba-Tercedor et al. } \\
\text { 2002; Bonada et al. 2002; Jáimez-Cuellar } \\
\text { et al., } 2002\end{array}$ & $\begin{array}{l}\text { hasta que no aparezcan nuevos taxones en las sucesivas redadas; protocolo } \\
\text { GUADALMED }\end{array}$ \\
\hline $\begin{array}{l}\text { Alba-Tercedor 1996; Hernández et al., } \\
\text { 1996, 1998; Solimini et al. 2000a,b }\end{array}$ & sustratos artificiales \\
\hline Prat et al., 1997 & $\begin{array}{l}\text { levantar 5-10 piedras en zonas con corriente y más en zonas con poca } \\
\text { corriente }\end{array}$ \\
\hline Hernández et al., 1996 & combinación red de mano y sustratos artificiales \\
\hline Rueda Sevilla et al, 1996 & exhaustivos muestreos con tamiz por todos los hábitats \\
\hline Munné \& Prat, 1997 & $\begin{array}{l}\text { unas } 10 \text { piedras en zonas reófilas y varias pasadas red por los márgenes y } \\
\text { zonas vegetación así como barrido con los pies en zonas flujo lenítico }\end{array}$ \\
\hline Rueda et al., 1998 & 10 submuestreos de $1 \mathrm{~m}$ con un cilindro de $30 \mathrm{~cm}$ de diámetro \\
\hline Bonada et al, 2000 & $\begin{array}{l}\text { red de mano intentando procurando muestrear todos los substratos } \\
\text { presentes con el mismo esfuerzo de muestreo }\end{array}$ \\
\hline $\begin{array}{l}\text { Solimini et al 2000a; Álvarez et al., 2001; } \\
\text { Martínez Mas et al., } 2004\end{array}$ & Red de mano 3 minutos \\
\hline $\begin{array}{l}\text { Younes et al., } 2002 \text { (BMWP); Iliopoulou- } \\
\text { Georgudaki } \text { et al., } 2003\end{array}$ & Surber \\
\hline $\begin{array}{l}\text { Jesús \& Formigo, 2001; Chaves et al., } \\
2001\end{array}$ & Red de mano 5 minutos \\
\hline Arluziaga, 2002 & Surber y red de mano durante 5-10 minutos \\
\hline Fernández et al., 2002 & Red de mano dos transectos de 10 minutos \\
\hline Gonçalves Moreira de Jesús, 2002 & 4 Surber distintos hábitats por tramo \\
\hline Gallardo-Mayenco et al., 2004 & $\begin{array}{l}\text { Red de mano en todos los mesohábitas, de } 2 \text { a } 5 \text { mangadas de } 30 \text { segundos } \\
\text { por mesohábitat. }\end{array}$ \\
\hline Ferreira et al., 2004 & Red de mano transecto $6 \mathrm{~m}$ en 3 minutos más 10 piedras \\
\hline Blijswijk et al, 2004 & Tamiz durante unos 20 minutos \\
\hline
\end{tabular}


de pie sobre el lecho del río, y obviamente tampoco con corrientes muy elevadas que puedan arrastrar a los técnicos.

\section{Valoración del IBMWP}

El IBMWP (antes BMWP') es una adaptación a la realidad ibérica del inglés BMWP (AlbaTercedor \& Sánchez-Ortega, 1988). En el índice original cada taxón tiene asociada una puntuación de acuerdo con su sensibilidad a la contaminación, siendo el valor del índice la suma de los valores de los taxones presentes (Armitage et al., 1983). El que sea un índice basado en el nivel taxonómico de familia (nivel recomendando en la norma de estandarización UNE-EN ISO 8689-1) ahorra costes temporales en su utilización, lo hace más sencillo, más práctico y aplicable geográficamente (Cao et al., 1997). Tanto el BMWP como el ASPT (la puntuación media de los taxones presentes que forman el BMWP) han sido profusamente empleados en el Reino Unido (Hawkes, 1997; Logan, 2001) y adaptados a las faunas de otras regiones (Alba-Tercedor \& Sánchez-Ortega, 1988; Chessman, 1995; Junqueira \& Campos, 1998; Benito de Santos \& Puig García, 1999; Mustow, 2002).

Las modificaciones originales de la adaptación ibérica fueron la inclusión de algunas familias y el cambio de puntuación de otras (AlbaTercedor \& Sánchez-Ortega, 1988). En un principio se presentó como una valoración de los taxones presentes en la comunidad, sin asociarla a ningún tipo concreto de muestreo (Alba-Tercedor \& Sánchez-Ortega, 1988) empleándose por vez primera con la red Surber (Alba-Tercedor \& Jiménez Millán, 1985). Posteriormente (AlbaTercedor, 1996) se ha indicado su utilización con muestras obtenidas con red manual sin tiempo definido (hasta que no se observen nuevos taxones en las redadas consecutivas), llegando a considerarse una particularidad del sistema ibérico que lo separa del original y del resto de adaptaciones (Alba-Tercedor, 2000).

La valoración del IBMWP se ha empleado, o propuesto su empleo, combinada con diversos métodos de muestreo (Tabla 3). Se trata de una valoración ampliamente utilizada en la
Península Ibérica, hasta el punto que se puede decir que es el índice de referencia para todos los estudios de macroinvertebrados de los ríos españoles (Prat, 2001). Resulta especialmente interesante a la hora de valorar el estado ecológico de un río según los requisitos de la Directiva Marco de Aguas, ya que valora la composición, diversidad y sensibilidad a la contaminación de los taxones de la comunidad de macroinvertebrados bentónicos (aunque no su abundancia).

La acotación de las distintas clases de estado ecológico debe de estar referida a los valores que obtendrían las estaciones no alteradas, de acuerdo con la DMA. En la definición original del IBMWP se establecieron también 5 clases (Alba-Tercedor \& Sánchez-Ortega, 1988), pero su transformación en las clases equivalentes de la DMA no debería ser directa, sino basada en los valores de esas estaciones hipotéticamente prístinas. En los años anteriores al desarrollo de este protocolo se observaron numerosos cambios en esas acotaciones, en ocasiones cambios de un río a otro y de año en año (Prat et al., 1997, 2000). En la cuenca del Ebro, Prat \& Munné (1999) realizaron una nueva acotación por regiones basada en la serie histórica de datos de macroinvertebrados de la Confederación Hidrográfica del Ebro. La precisión de dichos datos es bastante cuestionable, por lo que se decidió no emplear dicha acotación y mantener la original, toda vez que había estaciones que alcanzaban puntuaciones superiores a 100 en casi todas las regiones. Acotaciones recientes basadas en muestreos realizados con red manual mostraron una coincidencia casi total con las clases originales en los tipos de ríos que contaban con un número elevado de estaciones de referencia (Alba-Tercedor et al., 2002). Son necesarios nuevos estudios para mostrar la equivalencia o no de las clases de calidad al emplear un método u otro. No obstante, y hasta que se desarrolle una metodología predictiva efectiva para evaluar las diferencias entre las comunidades que se darían en ausencia de perturbación antrópica y las presentes en el tramo, o nuevos estudios indiquen lo contrario, se sigue con las clases originales. 


\section{Abundancias}

A la hora de definir las categorías de estado ecológico en los ríos según la fauna bentónica de invertebrados, la DMA (apartado 1.2.1 del anexo $\mathrm{V}$ ) hace referencia tanto a la composición como a la abundancia de los taxones. Así, disminuciones importantes de la densidad de macroinvertebrados debidas a causas antrópicas llevarán aparejada una reducción en la categoría de estado ecológico asignada. Este es un punto no recogido en la valoración del IBMWP, donde sólo se tiene en cuenta la presencia de cada taxón (Alba-Tercedor \& Sánchez-Ortega, 1988). El sistema de muestreo puede ser determinante a la hora de poder evaluar este punto, ya que sin el muestreo de una superficie concreta no es posible disponer de datos de densidades.

Para valorar esta reducción se deberán tener en cuenta las densidades de las comunidades presentes con anterioridad al impacto, si hubiera datos, y las de las comunidades no alteradas por efectos antrópicos del tipo de río de que se trate. Al realizar esta valoración, se deberá tener en cuenta la variabilidad que existe de forma natural en las comunidades. Fuentes naturales de variación importantes en cuanto a densidad de macroinvertebrados son los cambios significativos de caudal (riadas, estío), así como los ciclos vitales de los organismos (diferencias estacionales) (Gagneur, 1994; Gasith \& Resh, 1999; Argerich et al., 2004). Es necesaria una cierta experiencia, consultar la bibliografía existente sobre la zona y analizar los posibles cambios naturales entre muestras, antes de modificar la categoría de estado ecológico de una estación.

\section{Especies introducidas}

El impacto de las especies invasivas sobre las especies nativas, sus comunidades y ecosistemas ha sido ampliamente reconocido, y es considerado como un componente significativo del cambio global (Sakai et al., 2001), y de la reducción de la biodiversidad (Wilson, 2002), además de ser uno de los desafíos principales para la conservación en Europa en este milenio (Genovesi \& Shine, 2002). La introducción de especies alóctonas y la alteración del hábitat son las dos amenazas más importantes para la biodiversidad de las aguas continentales (Devin et al., 2005). En los ecosistemas de agua dulce, las especies introducidas causan la homogenización de las biotas, pudiendo tener efectos devastadores sobre las especies endémicas (Rahel, 2002; Englund, 2002; Brasher, 2003).

Si bien el impacto de estas especies sobre la comunidad de macroinvertebrados nativa en su conjunto puede ser valorado por los puntos anteriores, queda sin valorar su posible impacto sobre las especies recogidas en los Catálogos de Especies Amenazadas (estatal y autonómico). Su catalogación reconoce su estatus especial de conservación, y por ello deben valorarse los posibles impactos sobre estas especies particularmente.

Esta valoración determina la categoría de estado ecológico máxima a la que puede llegar un tramo que presente esas especies. La penalización aplicada es proporcional a los efectos negativos de cada especie, ya que resulta necesario distinguir entre las especies invasoras con efectos menores de aquellas con gran impacto (Parker et al., 1999). Se trata de una lista abierta a la inclusión de nuevas especies o al cambio de categoría de las ya presentes si estudios posteriores así lo aconsejan.

\section{Consideración final}

Este protocolo ha demostrado su aplicabilidad en los ríos aragoneses, permitiendo evaluar las comunidades de macroinvertebrados bentónicos. Las dos Surber en flujos distintos permiten realizar un muestreo multihábitat, y al estar basado en el muestreo de una superficie estandarizada permite las comparaciones espaciales (entre tramos) y temporales, proporcionando además datos de abundancia (individuos $/ \mathrm{m}^{2}$ ).

\section{AGRADECIMIENTOS}

El Departamento de Medio Ambiente del Gobierno de Aragón financió este proyecto (agradecemos especialmente la labor técnica de Paloma 
Barrachina). ATB disfrutó de una beca FICYT del Gobierno de Asturias. Juan Antonio Torralba, María Guzmán y Chesus Ferrer colaboraron en algunos de los trabajos de campo; José Alberto Martínez y Vanessa Prol en algunos de laboratorio.

\section{REFERENCIAS}

ALBA-TERCEDOR, J. 1996. Macroinvertebrados acuáticos y la calidad de las aguas de los ríos. IV Simposio del Agua en Adalucía (SIAGA), Almeria, 2: 203-213.

ALBA-TERCEDOR, J. 2000. BMWP', un adattamento spagnolo del British Biological Monitoring Working Party (BMWP) Score System. Biologia Ambientale, 14 (2): 65-67.

ALBA-TERCEDOR, J. y F. JIMÉNEZ MILLÁN. 1985. Evaluación de las variaciones estacionales de la calidad de las aguas del río Guadalfeo basada en el estudio de las comunidades de macroinvertebrados acuáticos y de los factores físico-químicos. En: Proyecto LUCDEME III.: 991. ICONA, Madrid.

ALBA-TERCEDOR, J. y A. SÁNCHEZ-ORTEGA. 1988. Un método rápido y simple para evaluar la calidad biológica de las aguas corrientes basado en el de Hellawell (1978). Limnetica, 4: 51-56

ALBA-TERCEDOR, J., P. JÁIMEZ-CUÉLLAR, M. ÁLVAREZ, J. AVILÉS, N. BONADA, J. CASAS, A. MELlADO, M. ORTEGA, I. PARDO, N. PRAT, M. RIERADEVALL, S. ROBLES, C. E. SÁINZ-CANTERO, A. SÁNCHEZ-ORTEGA, M. L. SUÁREZ, M. TORO, M. R. VIDALABARCA, S. VIVAS y C. ZAMORA-MUÑOZ. 2002. Caracterización del estado ecológico de ríos mediterráneos ibéricos mediante el índice IBMWP (antes BMWP'). Limnetica, 21 (3-4): 175-185.

ÁLVAREZ, M., I. PARDO, G. MOYÁ, G. RAMÓN \& A MARTÍNEZ-TABERNER. 2001. Invertebrate communities in temporary streams of the island of Majorca: a comparison of catchments with different land use. Limnetica, 20: 255-266.

ARGERICH, A. M. A. PUIG \& E. PUPILLI. 2004. Effect of floods of different magnitude on the macroinvertebrate communities of Matarranya stream (Ebro river basin, NE Spain). Limnetica, 23: 283-294.

ARMITAGE, P. D., D. MOSS, J. F. WRIGHT \& M. T. FURSE. 1983. The performance of a new bio- logical water quality score system based on macroinvertebrates over a wide range of unpolluted running-water sites. Water Res., 17: 333-347.

ARMITAGE, P. D., I. PARDO, M. T. FURSE \& J. F. WRIGHT. 1990. Assessment and prediction of biological quality. A demonstration to a British macroinvertebrate-based method in two Spanish rivers. Limnetica, 6: 147-156.

ARLUZIAGA, I. 2002. Variación de la calidad de las aguas de los ríos gipuzkoanos al cabo de veinte años (1981-2001). Munibe, 53: 39-56.

BARBOUR M. T., J. M. DIAMOND \& C. O. YODER. 1996. Biological assessment strategies: applications and limitations. En: Whole effluent toxiticy testing: an evaluation of methods and prediction of receiving system impacts. Gote \& al. (ed.): 245-270. Pellston, MI. Pensacola. USA.

BARBOUR, M. T., J. GERRITSEN, B. D. SNYDER, \& J. B. STRIBLING. 1999. Rapid Bioassessment Protocols for Use in Streams and Wadeable Rivers: Periphyton, Benthic Macroinvertebrates and Fish, Second Edition. EPA 841-B-99-002. U.S. Environmental Protection Agency; Office of Water; Washington, D.C. 339 pp.

BENITO DE SANTOS, G. y M. A. PUIG GARCÍA. 1999. BMWPC un índice biológico para la calidad de las aguas. adaptado a las características de los ríos catalanes. Tecnología del Agua, 191: 43-56.

BLIJSWIJK, W. V., C. N. COIMBRA \& M. A. S. GRAÇA. 2004. The use of biological methods based on macroinvertebrates to an Iberian stream (Central Portugal) receiving a paper mill effluent. Limnetica, 23: 307-314.

BONADA, N., M. RIERADEVALL y N. PRAT. 2000. Temporalidad y contaminación como claves para interpretar la biodiversidad de macroinvertebrados en un arroyo mediterráneo (Riera de Sant Cugat, Barcelona). Limnetica, 18: 81-90.

BONADA, N., N. PRAT, A. MUNNÉ, M. PLANS, C. SOLÁ, M. ÁlVAREZ, I. PARDO, G. MOYÀ, G. RAMÓN, M. TORO, S. ROBLES, J. AVILÉS, M. L. SUÁREZ, M. R. VIDAL-ABARCA, A. MELLADO, J. L. MORENO, C. GUERRERO, S. VIVAS, M. ORTEGA, J. CASAS, A. SÁNCHEZ-ORTEGA, P. JÁIMEZ-CUÉLLAR y J. ALBA-TERCEDOR. 2002. Intercalibración de la metodología GUADALMED. Selección de un protocolo de muestreo para la determinación del estado ecológico de los ríos mediterráneos. Limnetica, 21 (3-4): 13-33. 
BRASHER, A. M. D. 2003. Impacts of human disturbances on biotic communities in Hawaiian streams. BioScience, 53: 1052-1060.

CAO, Y., A. W. BARK \& W. P. WILLIAMS. 1997. Analysing benthic macroinvertebrate community changes along a pollution gradient: a framework for the development of biotic indices. Wat. Res., 31: 884-892.

CHAVES, M. L., P. M. CHAINHO, P. R. ALMEIDA, I. M. DOMINGOS, J. L. COSTA \& M. J. COSTA. 2001. Avaliaçao da qualidade da água com recurso à caracteruzaçao de comunidades biológicas nas bacias do Vouga, Mondego e Lis. En: Una cita europea con la Nueva cultura del Agua: la Directiva Marco, perspectivas en Portugal y España. II Congreso Ibérico sobre planificación y gestión del agua. N. Grande, P. Arrojo Agudo \& J. Martínez Gil (coord.): 1-12. Junta de Andalucía, Junta de Comunidades de CastillaLa Mancha, Fundación Calouste Gulbenkian, Universidad de Zaragoza, Institución Fernando el Católico, Zaragoza.

CHESSMAN, B. C. 1995. Rapid assessment of rivers using macroinvertebrates: a procedure based on habitat-specific sapling, family level identification and a biotic index. Australian Journal of Ecology, 20: 122-129.

DEVIN, S., L. BOLLACHE, P. \& NOËL \& J. N. BEISEL. 2005. Patterns of biological invasions in French freshwater systems by non-indigenous macroinvertebrates. Hydrobiologia, 551: 137-146.

ENGLUND, R. A. 2002. The loss of native biodiversity and continuing nonindigenous species introductions in Freshwater, Estuarine, and Wetland Communities of Pearl Harbor, Oahu, Hawaiian Islands. Estuaries, 25: 418-430.

FERNÁNDEZ, H. R., F. ROMERO, M. B. VECE, V. MANZO, C. NIETO y M. ORCE. 2002. Evaluación de tres índices bióticos en un río subtropical de montaña (Tucumán-Argentina). Limnetica, 21: 1-13.

FERREIRA, V., M. A. S. GRAÇA, M. J. FEIO \& C. MIEIRO. 2004. Water quality in the Mondego river basin: pollution and habitat heterogeneity. Limnetica, 23: 295-306.

GAGNEUR, J. 1994. Flash floods and drying up as major disturbances upon benthic communities in North-African wadis. Verth. Internat. Verein. Limnol., 25: 1807-1811.

GALLARDO-MAYENCO A. S. MACIAS y J. TOJA. 2004. Efectos de la descarga en la calidad del agua a lo largo de un río mediterráneo: el río Guadaira (Sevilla). Limnetica, 23(1-2): 65-78.

GASITH, A. \& V. H. RESH. 1999. Streams in Mediterranean climate regions: abiotic influences and biotic responses to predictable seasonal events. Ann. Rev. Ecol. Syst., 30: 51-81.

GENOMI, P. e L. STRADA. 2000. Confronto tra metodi di prelievo per l'analisi quantitative del macrobenthos. Biologia Ambientale, 14: 17-22.

GENOVESI, P. \& C. SHINE. 2002. European strategy on invasive alien species. T-PVS (2002) 8. Council of Europe, Estrasbrugo, 22 pp.

GONÇALVES MOREIRA DE JESUS, T. M. 2002. Impacto de centrais mini-hídricas na qualidad biológica da água: a "cascata” do Alova (Portugal). III Congreso Ibérico sobre Gestión y Planificación del Agua. La Directiva Marco del Agua: realidades y futuros. Sevilla, 13-17/11/2002: $12 \mathrm{pp}$.

HAWKES, H. A. 1997. Origin and development of the biological monitoring working party score system. Wat. Res., 32: 964-968.

HERNÁNDEZ, R., F. MARTÍNEZ, J. RUEDA, G. TAPIA y A. PUJANTE. 1996. Comparación de diferentes técnicas para el muestreo de macroinvertebrados en el río Magro (Valencia). Actas XII bienal RSEHN, Tomo extraordinario, 125 aniversario: $109-112$.

HERNÁNDEZ, R., J. RUEDA, G. TAPIA y F. MARTÍNEZ. 1998. Efectividad de los substratos artificiales para el muestreo de macroinvertebrados en ríos. Ecología, 12:151-166.

ILIOPOULOU-GEORGUDAKI, J. V. KANTZARIS, P. KATHARIOS, P. KASPIRIS, T. GEORGIADIS \& B. MONTESANTOU. 2003. An application of different bioindicators for assessing water quality: a case study in the rivers Alfeios and Pineios (Peloponnisos, Greece). Ecological Indicators, 2: 345-360.

JÁIMEZ-CUÉLLAR, P., S. VIVAS, N. BONADA, S. ROBLES, A. MELLADO, M. ÁllarEZ, J. AVILÉS, J. CASAS, M. ORTEGA, I. PARDO, N. PRAT, M. RIERADEVALL, C. E. SÁINZCANTERO, A. SÁNCHEZ-ORTEGA, M. L. SUÁREZ, M. TORO, M.R. VIDAL-ABARCA, C. ZAMORA-MUNOZZ y J. ALBA-TERCEDOR. 2002. Protocolo GUADALMED (PRECE). Limnetica, 21(3-4): 187-204.

JESÚS, T. \& N. FORMIGO. 2001. Estudo da qualidade biológica da água do rio Febros. En: Una cita europea con la Nueva cultura del Agua: la Directiva Marco, perspectivas en Portugal y España. II 
Congreso Ibérico sobre planificación y gestión del agua. N. Grande, P. Arrojo Agudo \& J. Martínez Gil (coord.): 1- 10. Junta de Andalucía, Junta de Comunidades de Castilla-La Mancha, Fundación Calouste Gulbenkian, Universidad de Zaragoza, Institución Fernando el Católico, Zaragoza.

JUNQUEIRA, V. M. \& S. C. M. CAMPOS. 1999. Adaptation of the "BMWP" method for water quality evaluation to Rio das Velhas watershed (Minas Gerais, Brazil). Acta Limnologica Brasiliensia, 10: 125-135.

KLEMM, D. J., P. A. LEWIS, F. FULK \& J. M. LAZORCHAT. 1990. Macroinvertebrate Field and Laboratory Methods for Evaluating the Biological Integrity of Surface Waters. U.S. Environmental Protection Agency, Washington, D.C, 256 pp.

LENAT, D. R. \& M. T. BARBOUR. 1994. Using benthic macroinvertebrate community structure for rapid, cost-effective, water quality monitoring: rapid bioassessment. En: Biological Monitoring of aquatic systems. S. L. Leb \& A. Spacie (eds.): 187215. Lewis publishers.

LOGAN, P. 2001. Ecological quality assessment of rivers and integrated catchment management in England and Wales. Journal of Limnology, 60(Suppl. 1): 25-32.

MARTÍNEZ, M., J. F. E. CORRECHER, A. PIÑÓN, M. A. MARTÍNEZ MURO y A. M. PUJANTE. 2004. Estudio del estado ecológico de los ríos de la cuenca hidrográfica del Júcar (España) mediante el índice BMWP'. Limnetica, 23: 331-346.

MUSTOW, S. E. 2002. Biological monitoring of rivers in Thailand: use and adaptation of the BMWP score. Hydrobiologia, 479: 191-229.

PARKER, I. M., D. SIMBERLOFF, W. M. LONSDALE, K. GOODELL, M. WONHAM, P. M. KAREIVA, M. H. WILLIAMSON, B. VON HOLLE, P. B. MOYLE, J. E. BYERS \& L. GOLDWASSER. 1999. Impact: toward a framework for understanding the ecological effects of invaders. Biological Invasions, 1: 3-19.

PLOTNIKOFF, R. W. 1994. Instream Biological Assessment Monitoring Protocols: Benthic Macroinvertebrates. Washington State Department of Ecology, Washington, D.C. 59 pp.

PRAT, N. 2001. Problemas y perspectivas en la definición del estado ecológico de los ecosistemas fluviales peninsulares ibéricos. En: Una cita europea con la Nueva Cultura del Agua: la Directiva Marco. Perspectivas en Portugal y España. II Congreso Ibérico sobre planificación y gestión del agua. N. Grande, P. Arrojo Agudo \& J. Martínez Gil (coords.): 1-15. Junta de Andalucía, Junta de Comunidades de Castilla La Mancha, Fundación Calouste Gulbenkian, Universidad de Zaragoza, Institución "Fernando el Católico". Zaragoza.

PRAT, N. y A. MUNNÉ. 1999. Objetivos de estado ecológico en los ríos de la cuenca del Ebro. Confederación Hidrográfica del Ebro, Zaragoza. $58 \mathrm{pp}$.

PRAT, N., M. RIERADEVALL, A. MUNNÉ, C. SOLÁ y G. CHACÓN. 1997. La qualitat ecológica del Besòs i el Llobregat. Informe 1996. En: Estudis de la qualitat ecològica dels rius, 2: 14132. Diputació de Barcelona, Barcelona.

PRAT, N., A. MUNNÉ, M. RIERADEVALL, C. SOLÀ y N. BONADA. 2000. ECOSTRIMED. Protocolo para determinar el estado ecológico de los ríos mediterráneos. Estudis de la qualitat ecològica dels rius, 8: 94 pp. Diputació de Barcelona, Barcelona.

PUJANTE, A., F. MARTÍNEZ-LÓPEZ y G. TAPIA. 1995. Macroinvertebrados y calidad de las aguas de los ríos próximos a la central térmica de Andorra (Teruel, España). Limnetica, 11(2): 1-8.

RAHEL, F. J. 2002. Homogenization of freshwater faunas. Annu. Rev. Ecol. Syst., 33: 291-315.

RESH, V. H., R. H. NORRI \& M. T. BARBOUR. 1995. Design and implementation of rapid assessment approaches for water resource monitoring using benhic macroinvertebrates. Australian Journal of Ecology, 20: 108-121.

ROSENBERG, D. M., I. J. DAVIES, D. G. COBB \& A. P. WIENS. 1997. Protocols for measuring biodiversity: Benthic Macroinvertebrates in Fresh Waters. Department of Fisheries and Oceans, Freshwater Institute, University Crescent, Winnipeg, Manitoba, 46 pp.

RUEDA, J., F. MARTÍNEZ, R. HERNÁNDEZ, C. LÓPEZ, A. PUJANTE, G. TAPIA y C. RODRÍGUEZ. 1996. El río Magro, calidad de sus aguas y caracterización de las comunidades de macroinvertebrados (NO de Valencia, España). Actas XII bienal RSEHN, Tomo extraordinario, 125 aniversario: $145-148$.

RUEDA, J., G. TAPIA, R. HERNÁNDEZ y F. MARTÍNEZ. 1998. El río Magro, parte I: evaluación de su calidad biológica mediante la aplicación del $\mathrm{BMWP}^{\prime}$ y del $\mathrm{ASPT}^{\prime}$. Ecología, 12: 135-150. 
SAKAI, A. K., F. W. ALLENDORF, J. S. HOLT, D. M. LODGE, J. MOLOFSKY, K. A. WITH, S. BAUGHMAN, R. J. CABIN, J. E. COHEN, N. C. ELSSTRAND, D. E. MCCAULEY, P. O'NEIL, I. M. PARKER, J. N. THOMPSON \& S. G. WELLER. 2001. The population biology of invasive species. Аnnu. Rev. Ecol. Syst., 32: 305-332.

SOLIMINI, A. G., P. GULIA, M. MONFRINOTTI \& G. CARCHINI. 2000a. Performance of different biotic indices and sampling methods in assessing water quality in the lowland stretch of the Tiber River. Hydrobiologia, 422/423: 197-208.

SOLIMINI, A. G., P. GULIA, M. MONFRINOTTI et G. CARCHINI. 2000b. Effet de différentes méthodes d'échantillonnage sur les valeurs d'indices biotiques de la qualité de l'eau du cours inférieur d'un fleuve méditerranéen: le Tibre. Annls. Limnol., 36: 135-143.
STOREY, A. W., D. H. D. EDWARD \& P. GRAZEY. 1991. Surber and kick sampling: a comparison for the assessment of macroinvertebrates community structure in streams of south-western Australia. Hydrobiologia, 211: 111-121.

TORRALBA BURRIAL, A. y F. J. OCHARAN. 2007. Comparación del muestreo de macroinvertebrados bentónicos fluviales con muestreador Surber y con red manual en ríos de Aragón (NE Península Ibérica). Limnetica, 26: 13-24.

YOUNES, Y., X. F. GARCÍA et J. GAGNEUR. 2002. Étude de l'impact des activités touristiques sur la qualité de l'eau et l'organisation des peuplements macrobenthiques au sein des cours d'eau de la Principauté d'Andorre. Revue des Sciences de l'Eau, 15: 421-424.

WILSON, E. O. 2002. El futuro de la vida. Galaxia Gutemberg-Círculo de Lectores, Barcelona, 317 pp. 